\title{
Permanent and transitory shocks in the presence of asymmetric error correction.
}

\section{Chan, $\mathrm{F} \quad$ and $\quad$ MacDonald $\mathrm{G}$ School of Economics and Finance, Curtin University}

Abstract: This paper highlights the potential importance of asymmetries in the loading vector of a set of cointegrated variables for the construction, analysis and interpretation of permanent and transitory shocks using impulse response functions. We derive an asymmetric version of the Permanent - Transitory decomposition suggested in Gonzalo and Ng (2001) and illustrate the potential importance of such asymmetries using the "cay" data of Lettau and Ludvigson (2004). 


\section{Introduction.}

This paper illustrates the sensitivity of impulse response functions and forecast error variance decompositions (FEVD's) to asymmetries in the loading vector of a cointegrated system. In particular we consider the impact of permanent and transitory (P-T) shocks using the method suggested in Gonzalo and $\mathrm{Ng}$ (2001). This procedure is, as noted in their paper, sensitive to the correct estimation of the loading vector $\gamma$ in the VECM, the role of which they refer to as "crucial" (pg 1543) since it (or rather its orthogonal complement $\gamma_{\perp}$ ) "defines the permanent shocks" (pg 1543). The idea that there may be non-linearities in the response of variables to the error correction term goes back to Granger and Lee (1989) who partitioned the error correction term into its positive and negative components in the dynamic equations of the VECM. Asymmetric responses of agents to positive and negative shocks (or gains and losses) is a potential source of variation in the loading vector and, since the work of Kahneman and Tversky (1979), has found an important place in the modeling of the behavior of economic agents. Clearly if this were the case then the (P-T) decomposition will be sensitive to the differential asymmetric responses of the VECM to positive and negative shocks. We illustrate the potential importance of such asymmetries using the consumption, wealth and income data of Lettau and Ludvigson (2004), which made a key contribution to our understanding of the role of wealth shocks on consumption. This is one area we might anticipate asymmetric responses with a literature stretching back to the famous Dusenberry (1949) "ratchet effect".

\section{Method.}

This paper aims to extend the result from Gonzalo and Ng (2001) to include Vector Error Correction Models with time varying loading vectors of the error correction term. Following the definition proposed in Gonzalo and Granger (1995), Gonzalo and Ng (2001) proposed a convenient method to identify the permanent and transitory shocks from a Vector Error Correction Model. By applying the Granger Representation Theorem, Gonzalo and Ng (2001) were able to identify two linear combinations that will extract the permanent and transitory shocks from the cointegrating system. The idea is to seek two linear combinations of the set of $I(1)$ variables such that the first combination will eliminate the transitory component and the second combination will eliminate the permanent component of the cointegrating system. However, their approach assumed that the loading vector is constant. Therefore, it is unclear if their approach is applicable when the cointegrating system exhibits asymmetric responses to the sign of the error correction term, that is when the short run adjustment of the system depends on 
the sign of the deviation from the long run equilibrium. The purpose of this section is to demonstrate that the idea of Gonzalo and Ng (2001) can be easily extended to such system.

Let $Y_{t} \sim I(1)$ be a $n$ 1vector of $I(1)$ variable and that there exists a $n r$ matrix, , such that $z_{t}=Y_{t} \sim I(0)$. The columns in represent $r<n$ cointegrating vectors. Let $L$ be the lag operator such that $L Y_{t}=Y_{t 1}$ and define the first difference operator $=\left(\begin{array}{ll}1 & L\end{array}\right)$, then by the Granger Representation Theorem, $Y_{t}$ has the following representation:

$$
Y_{t}={ }^{\prime} Y_{t 1}+(\mathrm{L}) Y_{t 1}+e_{t}
$$

where $\quad(L)=I_{n} \quad{ }_{i=1} L^{i} . \quad$ is the $n \quad r$ loading matrix where assumes to be constant over time. Equation (1) assumes the short run changes in $Y_{t}$, namely $Y_{t}$, responds symmetrically to $z_{t 1}$. That is, $Y_{t}$ reacts the same way to $z_{t 1}$ for both $z_{t 1}<0$ (system below the long run equilibrium) and $z_{t 1}>0$ (system above the long run equilibrium).

Note that since $Y_{t}$ is stationary, there exists a lag polynomial $\quad(L)$ such that

$$
Y_{t}=(L) e_{t}
$$

by the Wold's decomposition. Define and as the orthogonal complements of and , respectively such that ${ }^{\prime}={ }^{\prime}=0$, Gonzalo and Granger (1995) showed that $Y_{t}$ can be decomposed into permanent and transitory components, such that $Y_{t}=P_{t}+T_{t}$ where $P_{t} \sim I(1)$ and $T_{t} \sim I(0)$. Since $P_{t}$ is difference stationary, then by the Wold's Decomposition, there exists a moving average representations of both $P_{t}$ and $T_{t}$, such that 


$$
\left(\begin{array}{ll}
H_{11}(L) & H_{12}(L) \\
H_{21}(L) & H_{22}(L)
\end{array}\right)\left(\begin{array}{c}
P_{t} \\
T_{t}
\end{array}\right)=\left(\begin{array}{l}
u_{P t} \\
u_{T t}
\end{array}\right)
$$

Under the assumption that $\left({ }^{\prime}\right)^{1}$ and ()$^{1}$ exist, Gonzalo and Granger showed that $P_{t}={ }^{\prime}()^{1} \quad$ ' $Y_{t}$ and $T_{t}=\left({ }^{\prime}\right)^{1} Y_{t}=\left({ }^{\prime}\right)^{1} z_{t}$. In addition, if $H_{12}(1)=0$ then

$$
\lim _{h \rightarrow \infty} \frac{\partial E_{t}\left(Y_{t+h}\right)}{\partial u_{P t}} \neq 0
$$

and

$$
\lim _{h \rightarrow \infty} \frac{\partial E_{t}\left(Y_{t+h}\right)}{\partial u_{T t}}=0
$$

where $E_{t}(\bullet)$ denotes the expectation conditional on the information set at time $t$. Equations (4a) and (4b) define the permanent and transitory shocks, respectively. Specifically, the impact of $u_{P t}$ on the future values of $Y_{t}$ does not disappear whereas the impact of $u_{T t}$ on $Y_{t+h}$ diminishes as $h \rightarrow \infty$.

Define $G=(\quad, \quad)$, Proposition 1 in Gonzalo and $\mathrm{Ng}(2001)$ showed that $G e_{t}=\left(u_{P t}, u_{T t}\right)$. However, it is obvious that $u_{P t}$ and $u_{T t}$ are generally correlated as shown in equation (3). Gonzalo and $\mathrm{Ng}$ (2001) therefore proposed a linear transformation of $G e_{t}$, such that $H G e_{t}={ }_{t}=\left({ }_{P t},{ }_{T t}\right)$ with $\operatorname{Cov}\left({ }_{P t}, T_{t}\right)=0$ and that they satisfy the definitions of permanent and transitory shocks as defined in equations ( $3 a$ ) and (3b), respectively. That is

$\lim _{h \rightarrow \infty} \frac{\partial E_{t}\left(Y_{t+h}\right)}{\partial_{P t}} \neq 0$ and $\lim _{h \rightarrow \infty} \frac{\partial E_{t}\left(Y_{t+h}\right)}{\partial_{T t}}=0$. Note that the elements in $H$ are generally not identifiable without further restrictions. This is because any scalar multiple of $H$ can be used to orthogonalize $\left(u_{P t}, u_{T t}\right)$. For this reason, Gonzalo and $\mathrm{Ng}$ (2001) imposed the assumption that ${ }_{P t}$ and $T_{T t}$ are mutually and serially uncorrelated. This assumption, in addition to the restrictions implied by 
cointegration, provide enough conditions to identify $H$ uniquely. In fact, Gonzalo and Ng (2001) argued that the Choleski's decomposition could be a practical candidate for $H$. Under the assumption that the inverse of both $G$ and $H$ exist, $Y_{t}$ will then have a moving average representation in terms of ${ }_{t}$. That is, $\Delta Y_{t}=\tilde{\Theta}(L) \eta_{t}$ where $\tilde{\Theta}(L)=\Theta(L) G^{-1} H^{-1}$.

Now, consider the following asymmetric VECM model in the spirit of Granger and Lee (1989),

$$
Y_{t}=\left({ }^{+}+{ }^{*} s_{t 1}\right) z_{t 1}+(L) Y_{t 1}+e_{t}
$$

where $z_{t}=Y_{t}$ and $s_{t}$ denotes an indicator function such that

$$
s_{t}=\left\{\begin{array}{cc}
0 & z_{t} \geq 0 \\
1 & z_{t}<0 .
\end{array}\right.
$$

Unlike the VECM as defined in equation (1), the loading vector, , is no longer constant over time. Instead, it depends on the sign of the error correction term, $z_{t}=Y_{t}$. However, since $Y_{t}$ is stationary, it must have a moving average representation by the Wold's Decomposition theorem. Hence, there is a lag polynomial $(L)$ such that $Y_{t}=(L) e_{t}$. Moreover, let ${ }^{+}{ }^{*}$ and ${ }^{*}$ be the orthogonal complements to ${ }^{+}$and ${ }^{+}+{ }^{*}$, respectively, then it is clear that ${ }_{t}=\left({ }^{+}\left(\begin{array}{ll}1 & S_{t}\end{array}\right)+{ }^{*}{ }^{*} S_{t}\right)$ is the orthogonal complement to ${ }_{t}=\left({ }^{+}+{ }^{*} S_{t}\right)$. That is, ${ }_{t}{ }^{\prime}{ }_{t}=0$ for all $t$. Given these, Proposition 1 shows that $Y_{t}$ under equation (5) has a permanent-transitory decomposition as defined in Gonzalo and Granger (1995). 
Proposition 1': Let $Y_{t} \sim I(1)$ follows the dynamics as defined in equation (5). Assume that $\quad+\quad$ and $\left({ }^{+}+{ }^{*}\right)$ ' have no more than $n \quad r$ eigenvalues equal to 0 , then both $\left({ }^{+},\right)$and $\left({ }^{*},\right)$ are nonsingular. Moreover, let

$$
\left(\begin{array}{cc}
H_{11 t}(L) & H_{12 t}(L) \\
H_{21 t}(L) & H_{22 t}(L)
\end{array}\right)\left(\begin{array}{c}
P_{t} \\
T_{t}
\end{array}\right)=\left(\begin{array}{l}
u_{P t} \\
u_{T t}
\end{array}\right)
$$

be the autoregressive representation of $\left(Y_{t}, T_{t}\right)^{\prime}$, with $H_{12 t}(1)=0$ for all $t$ and the coefficient matrix satisfies the stationarity condition as stated in Nicholls and Quinn (1981), then $Y_{t}$ has a permanenttransitory decomposition $Y_{t}=P_{t}+T_{t}$ where

$$
P_{t}=\left(t_{t}^{\prime}\right)^{1}{ }_{t}{ }^{\prime} Y_{t}
$$

and

$$
T_{t}={ }_{t}\left({ }_{t}\right){ }^{1} z_{t} \text {. }
$$

Proposition (2) contains the extension of Gonzalo and Ng (2001) decomposition to processes as defined in equation (5).

Proposition 2: Let $G_{t}=\left({ }_{t},{ }^{\prime}\right)^{\prime}$, then $G_{t} e_{t}=\left(u_{P t}, u_{T t}\right)$. Moreover, let $H_{t}{ }^{1}$ denotes the Choleski decomposition of $G_{t} e_{t}$ at time $t$, then $H_{t} G_{t} e_{t}={ }_{t}=\left(\begin{array}{ll}{ }_{P t}, & { }_{T t}\end{array}\right)^{\prime}$ such that the elements in ${ }_{t}$ are

\footnotetext{
${ }^{1}$ Proofs for proposition 1 and Proposition 2 are in Appendix 1.
} 
serially and mutually uncorrelated with $T_{P t}$ and $T_{T t}$ satisfy the definitions of permanent and transitory shocks as defined in equations (4a) and (4b).

\section{Application.}

To illustrate the potential importance of asymmetries, we use the original consumption, wealth and income (cay) data from Lettau and Ludvigson $(2004)^{2}$. Their paper found evidence of a single cointegrating vector between the cay variables and they then applied the Gonzalo and Ng (2001) decomposition to separate the permanent and transitory shocks. Importantly, wealth was the only variable for which the loading parameter was significant and their results showed that most of the shocks to wealth were of a transitory nature and that these transitory shocks were relatively persistent. In this section, we re-estimate their model and demonstrate the potential importance of asymmetry in identifying permanent and transitory shocks as well as analyzing variance decomposition.

Table 1 shows the estimated parameters of the symmetric and asymmetric VECM ${ }^{3}$ with a single lag. Both the lag order of the VECM and the cointegrating vector were selected to replicate the original results of Lettau and Ludvigson (2004) and thus provide a contrast to the asymmetric case. In the symmetric case, the estimated loading coefficients are significant only in the dynamic equation for wealth ${ }^{4}$. Once we allow for asymmetry, whilst the parameters on the dynamic terms in the VECM are largely unchanged, the loading coefficient is now significant (with expected sign) in the dynamic equation for consumption when the ect term is positive and significant in the dynamic equation for wealth when the ect term is negative. One possible rationalization for these results is that they are a simple reflection of liquidity constraints. Consumers find it easier to reduce consumption when above equilibrium but are liquidity constrained when below equilibrium, and hence, consumption plays no part in the error correction.

If we follow Gonzalo and Ng's advice and set the insignificant parameters in the loading vector to zero

then the $G$ matrix becomes very simple. In the symmetric case, $G$ will select the consumption errors as the first permanent shocks (P1) and income errors as the second (P2). In the asymmetric case the first permanent shock (P1) will be based on the wealth errors when $\hat{u}_{t}>0$ and on the consumption errors

2 The data spans from 1951:4 to $2003: 1$ and is available from Martin Lettau's web page at http://faculty.haas.berkeley.edu/lettau/data cay.html

3 The cointegrating vector was $(1,-0.2615,-0.6239)$ for $(c, a, y)$.

${ }^{4}$ Tests of zero restrictions on the elements of the loading vector are in footnotes below Table 1 
when $\hat{u}_{t}<0$ In both case the second permanent shock (P2) will be based on the income errors. In both the symmetric and asymmetric cases, the transitory shock $(T)$ will be based on the cointegration vector weights. Table 2 shows the $G$ matrix.

Table 1. Parameter Estimates in VECM

\begin{tabular}{|l|l|l|l|l|l|l|l|}
\hline & \multicolumn{3}{|l|}{ Symmetric VECM } & \multicolumn{3}{l|}{ Asymmetric VECM } \\
\hline & $\Delta c_{t}$ & $\Delta a_{t}$ & $\Delta y_{t}$ & $\Delta c_{t}$ & $\Delta a_{t}$ & $\Delta y_{t}$ \\
\hline$\Delta c_{t-1}$ & 0.203 & 0.064 & 0.499 & 0.197 & 0.055 & 0.493 \\
& $(2.66)$ & $(0.18)$ & $(3.20)$ & $(2.62)$ & $(0.16)$ & $(3.16)$ \\
\hline$\Delta a_{t-1}$ & 0.043 & 0.099 & 0.090 & 0.044 & 0.100 & 0.091 \\
& $(2.70)$ & $(1.37)$ & $(2.78)$ & $(2.84)$ & $(1.40)$ & $(2.83)$ \\
\hline$\Delta y_{t-1}$ & 0.067 & -0.041 & -0.123 & 0.058 & -0.053 & -0.132 \\
& $(0.04)$ & $(-0.23)$ & $(-1.50)$ & $(1.46)$ & $(-0.29)$ & $(-1.60)$ \\
\hline$\hat{u}_{t 1}$ & $-0.036^{\mathrm{a}}$ & 0.387 & $0.008^{\mathrm{a}}$ & & & & \\
& $(-1.38)$ & $(3.24)$ & $(0.16)$ & & -0.178 & $0.209^{\mathrm{b}}$ & $-0.119^{\mathrm{b}}$ \\
$\hat{u}_{t 1}(1$ & & & & $(-3.19)$ & $(0.81)$ & $(-1.03)$ \\
\hline$\left.\hat{u}_{t 1_{1}{ }_{t} s_{1}}\right)$ & & & & $\begin{array}{l}0.061^{\mathrm{b}} \\
(1.43)\end{array}$ & $\begin{array}{l}0.510 \\
(2.58)\end{array}$ & $\begin{array}{l}0.097^{\mathrm{b}} \\
(1.09)\end{array}$ \\
\hline $\bar{R}^{2}$ & & & & 0.19 & 0.04 & 0.09 \\
\hline
\end{tabular}

$\mathrm{t}$ statistics in parenthesis () below estimated coefficients. For the symmetric VECM a test of the null that the 2 coefficients on the ect term with "a" superscripts were jointly zero had a p-value of 0.25 , in the asymmetric VECM the test of the null that the 4 coefficients on the ect terms with " $b$ " superscripts were jointly zero yielded a p-value of 0.44 .

Since the PT errors are constructed as $G^{*}$, it is clear that:

1. The construction of the transitory shock will be unaffected and how much it impacts on each of the variables will depend on the loading vectors.

2. The second Permanent shock will be the same in the two decompositions.

3. All the differences will come from the $1^{\text {st }}$ permanent shock as this will differ according to whether the error correction term is positive or negative. 
Figure 1 show the IRF's from the symmetric case and the "positive" asymmetric case ${ }^{5}$ and as can be seen these show differing adjustment dynamics in the face of a transitory shock. In the first diagram (symmetric) the positive transitory shock shows up largely as a fall in wealth which pushes the ect positive, whereas in the second case (positive asymmetric) it is consumption which jumps upwards, generating the positive shock. As noted above the dynamics of the VECM's are very similar in both cases and the differing adjustment structure highlights the importance of the asymmetries in the loading vector.

Table $2^{6}$. Full PT Decompositions

\begin{tabular}{|l||l|l|l||l|l|l||l|l|l|}
\hline \multicolumn{1}{|c||}{} & \multicolumn{3}{c||}{ Symmetric VECM } & \multicolumn{3}{c||}{$\begin{array}{c}\text { Asymmetric VECM } \\
u_{t}>0\end{array}$} \\
\hline & C & A & Y & C & A & Y & \multicolumn{3}{c|}{$\begin{array}{c}\text { Asymmetric VECM } \\
u_{t}\end{array}$} \\
\hline P1 & 1 & 0 & 0 & 0 & 1 & 0 & 1 & 0 & 0 \\
\hline P2 & 0 & 0 & 1 & 0 & 0 & 1 & 0 & 0 & 1 \\
\hline T & 1 & -0.2615 & -0.6239 & 1 & -0.2615 & -0.6239 & 1 & -0.2615 & -0.6239 \\
\hline
\end{tabular}

Table 3. Variance Decompositions

\begin{tabular}{|c|c|c|c|c|c|c|c|c|c|c|c|c|}
\hline \multirow[b]{3}{*}{ Horizon } & \multicolumn{6}{|c|}{ Symmetric } & \multicolumn{6}{|c|}{ Asymmetric Positive } \\
\hline & \multicolumn{2}{|c|}{ Consumption } & \multicolumn{2}{|c|}{ Wealth } & \multicolumn{2}{|c|}{ Income } & \multicolumn{2}{|c|}{ Consumption } & \multicolumn{2}{|c|}{ Wealth } & \multicolumn{2}{|c|}{ Income } \\
\hline & $\mathrm{P}$ & $\mathrm{T}$ & $\mathrm{P}$ & $\mathrm{T}$ & $\mathrm{P}$ & $\mathrm{T}$ & $\mathrm{P}$ & $\mathrm{T}$ & $\mathrm{P}$ & $\mathrm{T}$ & $\mathrm{P}$ & $\mathrm{T}$ \\
\hline 1 & 1 & 0 & 0.976 & 0.09024 & 1 & 0 & 0.1582 & 0.8418 & 1 & 0 & 1 & 0 \\
\hline 2 & 0.9708 & 0.0292 & 0.1098 & 0.8902 & 0.9677 & 0.0323 & 0.2516 & 0.74784 & 0.9994 & 0.0006 & 0.9275 & 0.0725 \\
\hline 3 & 0.9679 & 0.0321 & 0.1123 & 0.8877 & 0.9674 & 0.0326 & 0.2713 & 0.7287 & 0.9993 & 0.0007 & 0.9275 & 0.0725 \\
\hline 4 & 0.9679 & 0.0321 & 0.1145 & 0.8855 & 0.9674 & 0.0326 & 0.2779 & 0.7221 & 0.9993 & 0.0007 & 0.9272 & 0.0728 \\
\hline Inf. & 0.9652 & 0.0348 & 0.1215 & 0.8785 & 0.9653 & 0.0347 & 0.2851 & 0.7149 & 0.9993 & 0.0007 & 0.9236 & 0.0764 \\
\hline
\end{tabular}

Finally, Table 3 shows the orthogonalized variance decomposition. As in Lettau and Ludvigson ${ }^{7}$, transitory shocks dominate wealth in the symmetric case, with $88 \%$ of the variation due to such shocks whilst consumption and income are largely driven by the two permanent shocks. In the asymmetric

\footnotetext{
${ }^{5}$ Because of the construction of the $G$ matrix the asymmetric negative case is essentially the same as the symmetric one and is therefore omitted for brevity.

${ }^{6}$ Note that we chose to retain the order cay, results are basically the same for other orderings.

${ }^{7}$ The results differ slightly as we retained the cay ordering.
} 
case, however, the results suggest it is consumption, which is driven more by transitory shocks with $71 \%$ of the variation being attributable to the transitory shock and only $29 \%$ to the two permanent shocks. Wealth, on the other hand, is now driven almost exclusively by the two permanent shocks.

Figure 1 Impulse Response Functions
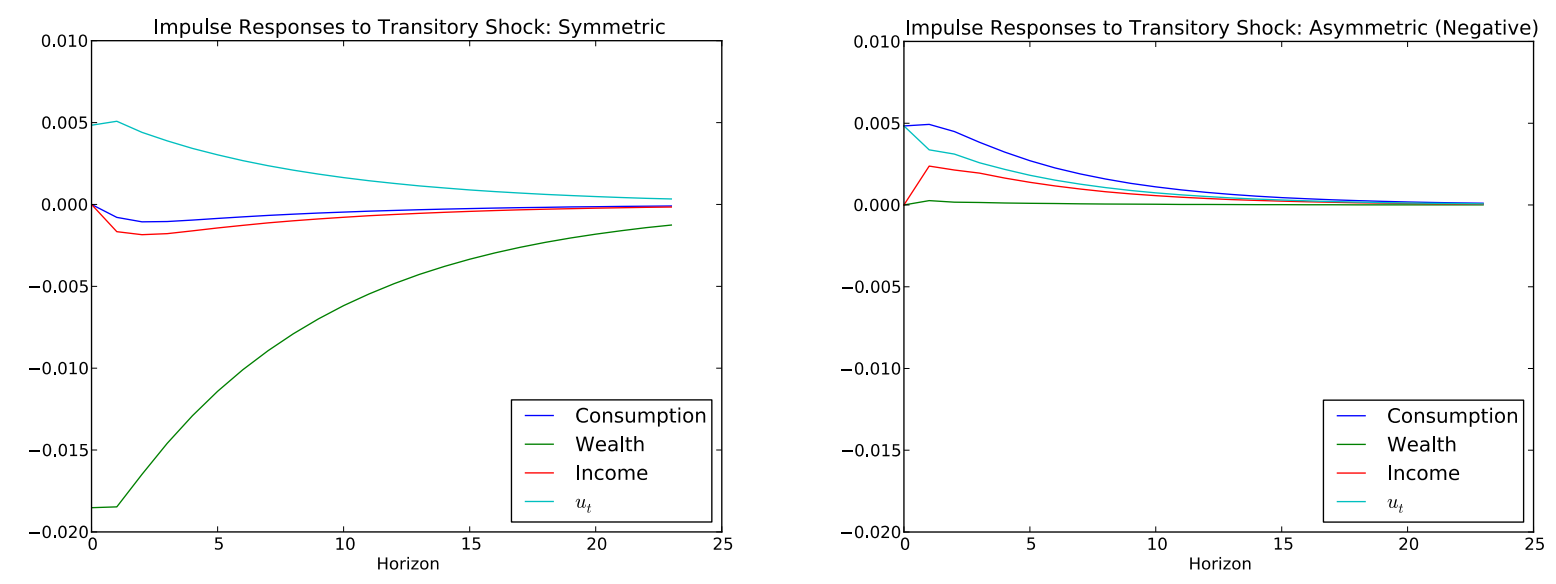

\section{Conclusion}

This short paper illustrates the importance of asymmetric behavior in the VECM model for permanent transitory decompositions. In particular, in Section 2, we derive the specification of a model of an appropriate permanent-transitory decomposition under the assumption of asymmetry in the loading vector. The model follows closely that of Granger and Gonzalo (1995) and Gonzalo and Ng (2001) and has the advantage, for the applied worker, of being simple to implement. We provide a practical illustration of the sensitivity of such decompositions to asymmetries in the loading vector and illustrate their practical importance for variance decompositions and impulse responses using the cay data of Lettau and Ludvigson (2004). Note that the asymmetric behaviour appears only in the adjustment process (the loading vectors) and yet it has important implications for both permanent-transitory shock decompositions and the impulse responses. Given the emphasis often placed on impulse responses and variance decompositions in models using the VECM framework, and their importance in drawing conclusions for the macroeconomic policy implications of such models, it is crucial to note the sensitivity of the results to the parameterization of the loading vector and to the potential for asymmetric responses to significantly alter the conclusions of such analysis. 


\section{$\underline{\text { References }}$}

Dusenberry, J.S. (1949) Income, Saving and the Theory of Consumer Behaviour, Cambridge: Harvard University Press.

Gonzalo, J. and Granger, C.W.J., (1995) Estimation of Common Long-memory Components in Cointegrated Systems" Journal of Business and Economic Statistics, American Statistical Association, Vol. 13(1), pp. 27-35.

Gonzalo, J. and Ng, S. (2001) "A Systematic Framework for Analyzing the Dynamic Effects of Permanent and Transitory Shocks", Journal of Economic Dynamics and Control, 25(10), 1527-1546.

Granger, C.W.J. and Lee, T.H. (1989) "Investigation of Production, Sales and Inventory Relationships Using Multicointegration and Non-Symmetric Error Correction Models", Journal of Applied Econometrics, 4, S145-S159.

Kahneman, D. and Tversky, A. (1979) "Prospect Theory: An Analysis of Decision under Risk", Econometrica, XLVII, 263-291.

Lettau, M. and Ludvigson, S.C. (2004) "Understanding Trend and Cycle in Asset Values: Reevaluating the Wealth Effect on Consumption", American Economic Review, 94, 276-299.

Nicholls, D.F. and B.G. Quinn (1981). "The estimation of multivariate random coefficient autoregressive models", Journal of Multivariate Analysis, 11(4) pp 544-555.

Shin, Y., Yu, B. and Greenwood-Nimmo, C. (2011) "Modelling Asymmetric Cointegration and Dynamic Multipliers in a Nonlinear ARDL Framework", working paper downloaded at http://ssrn.com/abstract=1807745. 


\section{Appendix 1}

Proof of Proposition 1: Under assumptions and Proposition 3 in Gonzalo and Granger (1995), $Y_{t}$ can be expressed as follows at any given $t$ :

$$
Y_{t}=\left\{\begin{array}{lll}
(+, \quad)^{1}+Y_{t}+\left({ }^{+}+\right)^{1} z_{t} & z_{t} \geq 0 \\
\left({ }^{*}\right){ }^{1} * Y_{t}+(,+)^{1} z_{t} & z_{t}<0 .
\end{array}\right.
$$

Combining the two expressions on the right hand side with the indicator function as defined in equation (6) gives the expressions for $P_{t}$ and $T_{t}$. Moreover, since the coefficient matrix in the autoregressive representation is invertible, there exists a moving average representation for $\left(P_{t}, T_{t}\right)$ 'at any given $t$ such that

$$
\left(\begin{array}{c}
P_{t} \\
T_{t}
\end{array}\right)=\left(\begin{array}{ll}
H_{t}^{11}(L) & H_{t}^{12}(L) \\
H_{t}^{21}(L) & H_{t}^{22}(L)
\end{array}\right)\left(\begin{array}{c}
u_{P t} \\
u_{T t}
\end{array}\right) .
$$

Under the assumption that $H_{12 t}(1)=0$ for all $t$, it implies that $H_{t}^{12}(1)=0$ for all $t$. Thus, a straightforward application of Proposition 1 in Gonzalo and Granger (1995) shows that $\left(u_{P t}, u_{T t}\right)$ satisfy the definitions of permanent and transitory shocks as defined in equations (4a) and (4b), respectively. This completes the proof.

Proof of Proposition 2: The proof is similar to the proof of Proposition 1 in Gonzalo and Ng (2001) by replacing with $t$. This completes the proof. 